\title{
Distribution across Age of Raw and Scaled Intelligence Scores - the DARSIS model
}

\author{
Y. Cramer ${ }^{1 *}$
}

October 15, 2021

${ }^{1}$ Department of Research and Development, SCALIQ

* Corresponding author, email: y.cramer@scaliq.com

\begin{abstract}
The mean raw score on intelligence tests rises steeply in childhood before stabilizing for adolescents and adults, Little is known however about how the different percentiles, let alone the entire raw score distribution, change from early childhood to adulthood. This study will show that there is a regular, mathematical relationship between age, raw scores and scaled scores on intelligence test subtests with a high $g$-loading. Using the norm tables from 60 subtests from 19 different intelligence tests normed in 5 different countries between 1984-2020, a relatively simple model with three coefficients and a variable 'difficulty' parameter is shown to explain almost all of the variance in the norm tables. Smaller errors are found for the mean of norm tables, and for higher performance, but not for greater age. The year in which a test was normed also did not appear to influence the fit of the model. Possible applications of the DARSIS model, such as the creation of norms for above-level testing, creating extended norms, a reduced parameter space when norming new intelligence tests and calculating reference ages are discussed.
\end{abstract}

\section{keywords}

intelligence; norms; IQ-tests; above-level testing;

\section{Introduction}

Ever since intelligence tests are normed for multiple age groups, researchers have noticed that the raw score distribution changes gradually across age. On average, older children and adults perform better (attain higher raw scores) than younger children. In practically all test standardizations, the mean raw score for heavily g-loaded subtests rises gradually with increasing age and stabilizes somewhere around age 15-25. (Li et al., 2004; Tucker-Drob, 2009; McArdle, Ferrer-Caja, Hamagami, \& Woodcock, 2002; Hartshorne \& Germine, 2015). These studies are about the average score however, corresponding to the 50th percentile, while the variability of scores on intelligence tests is quite large. For example, in the Dutch standardization of the WISC-V, the top 10\% of 8-year-olds outperforms the bottom $10 \%$ of 16 -year-olds in their performance on the matrices subtest.

In contrast to the change in mean scores as mentioned above, little is known about how the entire score distribution changes across age. In this paper, the aim is to model how raw intelligence test 
scores change across the age and ability spectrum by modeling the relationship between age, raw score and scaled scores using a continuous function with a variable difficulty parameter.

\section{Data}

In order to model how the raw score distribution of intelligence tests changes from early childhood into adulthood, norm tables from several different intelligence tests were used as data. Norm tables from intelligence tests needed to be available to the researcher and adhere to the following inclusion criteria to be included in this study:

1. Norms needed to be from nationally standardized intelligence tests. Norms from dynamic tests, 'learning ability' tests and other unstandardized or unnormed tests were not included.

2. At least part of the norms needed to concern children in the range of 0-20 years old.

3. Norms needed to be available for more than one age group. So for example, an intelligence test with only one norm table for 9-year-old's was not included, as no change in raw scores across age can be observed.

4. Norms needed to be detailed enough such that for each raw score, a scaled score or percentile was given for each available age band (excluding floor- and ceiling-effects). Norms such as some Raven Matrices norms that only indicated which raw scores corresponded to the the 5th, 10th, 25th, 50th, 75th, 90th and 95th percentile were therefore excluded.

5. For tests with multiple subtests, the 2, 3 or 4 most highly g-loaded subtests were chosen, by looking at the correlation between the subtest and the Total IQ score and the results of factor analyses when available.

Over the course of several years, intelligence test norms were gathered and digitalized. Norms from the following tests were included:

WISC-V-NL The Wechsler Intelligence Scale for Children, fifth edition for the Netherlands is an individually administered intelligence test for children aged 6;0 to 16;11. It consists of 14 subtests, of which 7 are used to calculate the Full Scale IQ score. The WISC-V-NL was normed in 2017 for the entire Dutch-speaking area (Netherlands \& Flanders) using a representative sample of $n=1200$ children. Subtest norms selected for this study include matrices (MA), figure weights (GW) and similarities $(\mathrm{OV})$.

WISC-V-EN The Wechsler Intelligence Scale for Children, fifth edition Extended Norms are special norms designed to be used to more clearly identify highly gifted children. They were developed using the normative sample from the USA, along with the data of 108 gifted children for validity purposes. Subtest norms selected for this study include matrices (MA), visual puzzles (VP) and picture concepts $(\mathrm{PC})$.

WISC-IV-EN The Wechsler Intelligence Scale for Children, fourth edition Extended Norms are special norms designed to be used to more clearly identify highly gifted children. They were developed using the normative sample from the USA, along with the data of 157 gifted children for validity purposes. Subtest norms selected for this study include matrices (MA), figure weights (FW), visual puzzles (VP) and picture concepts (PC). 
WISC-III-NL The Wechsler Intelligence Scale for Children, third edition for the Netherlands is an individually administered intelligence test for children aged 6;0 to 16;11. It consists of 13 subtests, of which 10 are used to calculate the Full Scale IQ score. The WISC-III-NL was normed in 2005 for the entire Dutch-speaking area (Netherlands \& Flanders) using a representative sample of $n=1239$ children. Subtest norms selected for this study include similarities (OV) and arithmetic (RE).

WISC-R-NL The Wechsler Intelligence Scale for Children, revised edition for the Netherlands is an individually administered intelligence test for children aged 6;0 to $16 ; 11$. It consists of 12 subtests, all of which are used to calculate the Full Scale IQ score. The WISC-R-NL was normed in 1986 for the entire Dutch-speaking area (Netherlands \& Flanders) using a representative sample of $n=1961$ children. Subtest norms selected for this study include similarities (OV) and block design (BP).

RAKIT-2 The Revisie Amsterdamse Kinder Intelligentie Test, second edition is a Dutch individually administered intelligence test for children aged $4 ; 0$ to $12 ; 5$. It consists of 12 subtests, all subtests combined are used to calculate the Full Scale IQ score. The RAKIT- 2 was normed in the Netherlands in 2012 using a representative sample of $n=1424$ children. Subtest norms selected for this study include analogies (AN), exclusion (EX) and quantities (KW).

RAKIT The Revisie Amsterdamse Kinder Intelligentie Test is a Dutch individually administered intelligence test for children aged 4;2 to 10;11. It consists of 12 subtests, all subtests combined are used to calculate the Full Scale IQ score. The RAKIT was normed in the Netherlands in 1984 using a representative sample of $n=1415$ children. Subtest norms selected for this study include analogies (AN), exclusion (EX) and quantities (KW).

GOS-2.5-4.5 The Groninger Ontwikkelings-Schalen is a Dutch, individually administered intelligence test for children aged 2;6 to 4;6. It consists of 13 subtests, all subtests combined are used to calculate the Full Scale IQ score. The GOS was normed in the Netherlands in 1996 using a representative sample of $n=982$ children. Subtest norms selected for this study include magic disk (MS) and vocabulary (WS).

WPPSI-IV-NL The Wechsler Preschool and Primare Scale of Intelligence, fourth edition for the Netherlands is an individually administered intelligence test for children aged 2;6 to 6;11. It consists of 15 subtests, of which 5 are used to calculate the Full Scale IQ score. The WPPSI-IV-NL was normed in 2020 for the entire Dutch-speaking area (Netherlands \& Flanders) using a representative sample of $n=997$ children. Subtest norms selected for this study include matrices (MA), word reasoning (WR) and picture concepts (PC).

WPPSI-III-NL The Wechsler Preschool and Primary Scale of Intelligence, third edition for the Netherlands \& Flanders is an individually administered intelligence test for children aged $2 ; 6$ to $7 ; 11$. It consists of 14 subtests, of which 7 are used to calculate the Full Scale IQ score ( 4 subtests for the younger ages). The WPPSI-III-NL was normed in 2009 for the entire Dutch-speaking area (Netherlands \& Flanders) using a representative sample of $n=1148$ children. Subtest norms selected for this study include matrices (MA), word reasoning (WR) and picture concepts $(\mathrm{PC})$.

COVAT-CHC The COgnitieve VAardigheidsTest volgens het CHC-model is a Flemish group-administered intelligence test for children aged 9;6 to 13;11. It consists of 8 subtests, of which all are used to calculate the Full Scale IQ score. The COVAT-CHC was normed in 2014 in Flanders using a representative sample of $n=3071$ children. Subtest norms selected for this study include point sequences (PR), figure sequences (FR), opposites (TE) and folding boxes (DP). 
KAIT-NL The Kaufman Adult Intelligence Test, Dutch edition is an individually administered intelligence test for individuals aged 14;0 to 84;11. It consists of 10 subtests, of which 6 are used to calculate the Full Scale IQ score. The KAIT-NL was normed in 2003 for the entire Dutchspeaking area (Netherlands \& Flanders) using a representative sample of $n=990$ adolescents and adults. Subtest norms selected for this study include symbol learning (SL), logical reasoning (LR) and secret codes (GC).

SON-R 2.5-7-NL The Snijders-Oomen Nonverbal 2.5-7 is a nonverbal, individually administered intelligence test for children aged 2;0 to 7;11. It consists of 6 subtests, all of which are used to calculate the Full Scale IQ score. The SON-R 2.5-7-NL was normed in the Netherlands in 1993 using a representative sample of $n=1124$ children. Subtest norms selected for this study include analogies $(\mathrm{AN})$ and categories $(\mathrm{CA})$.

SON-R 2.5-7-NL-DE The Snijders-Oomen Nonverbal 2.5-7 is a nonverbal, individually administered intelligence test for children aged 2;0 to 7;11. It consists of 6 subtests, all of which are used to calculate the Full Scale IQ score. The SON-R 2.5-7 was normed in the Netherlands in 1993 and in the Netherlands and Germany in 2010 using representative samples of $n=1124$ and $n=2148$ children respectively. Combined norms were created. Subtest norms selected for this study include analogies (AN) and categories (CA).

SON-R 6-40 The Snijders-Oomen Nonverbal 6-40 is a nonverbal, individually administered intelligence test for individuals aged $6 ; 0$ to $39 ; 11$. It consists of 4 subtests, which are combined to calculate the Full Scale IQ score. The SON-R 6-40 was normed in the Netherlands and Germany in 2010 using a representative sample of $n=2000$ children and adults. Subtest norms selected for this study include analogies (AN) and categories (CA).

WAIS-III-NL The Wechsler Adult Intelligence Scale, third edition for the Netherlands is an individually administered intelligence test for adults aged 16;0 to 84;11. It consists of 14 subtests, of which 6 are used to calculate the Full Scale IQ score. The WAIS-III-NL was normed in 2003 for the entire Dutch-speaking area (Netherlands \& Flanders) using a representative sample of $n=670$ adults. Subtest norms selected for this study include matrices (MA) and similarities (OV).

WAIS-IV-NL The Wechsler Adult Intelligence Scale, fourth edition for the Netherlands is an individually administered intelligence test for adults aged 16;0 to 84;11. It consists of 12 subtests, of which 6 are used to calculate the Full Scale IQ score. The WAIS-IV-NL was normed in 2003 for the entire Dutch-speaking area (Netherlands \& Flanders) using a representative sample of $n=1510$ adults. Subtest norms selected for this study include matrices (MA), similarities $(\mathrm{OV})$, figure weights (GW) and visual puzzles (FS).

WNV-NL The Wechsler Non-Verbal for the Netherlands is an individually administered non-verbal intelligence test for children and adults aged 4;0 to 20;11. It consists of 6 subtests, of which 4 are used to calculate the Full Scale IQ score. The WNV-NL was normed in 2008 using a representative sample of $n=1720$ children and adults. Subtest norms selected for this study include matrices (MA), object assembly (FL) and spatial span (RO).

RAVEN The Raven matrices is a set of non-verbal intelligence tests for children and adults. Multiple versions exist, including the Colored Progessive Matrices (CPM), the Standard Progressive Matrices (SPM) and the Advanced Progressive Matrices (APM). Norms used in this study are from the standardization of the SPM in 1984 in the United kingdom. 


\subsection{Data preparation}

All test norms were digitalized and entered into data frames in R. All scaled scores (or percentiles) were transformed to their corresponding $z$-scores on the standard-normal distribution $(\mu=0, \sigma=1)$. Since the purpose of this study is to model the change in the raw score distributions from early childhood to adulthood, and not the decline in cognitive ability that is associated with older age, norm tables were included upto the age where the raw score associated with average performance (a $z$-score of 0 ) showed a declining trend for older ages. For example, if in a subtest norm a raw score of 49 was associated with a $z$-score of 0 for 16-17-year-olds, 18-19-year-olds and 20-24-year-olds, but with a $z$-score of 0.33 for 25-30-year-olds, the norms for age 25 and up would not be included.

\subsubsection{Floor and ceiling-effects}

Some norm tables featured quite extensive floor- and ceiling-effects. In the data preparation, some entries were therefore removed. A norm table entry with a floor effect is defined as the lowest possible scaled score within an age band for which there exists, in the same age band, a raw score that is higher that yields the same scaled score. A norm table entry with a ceiling effect is defined as the highest possible scaled score within an age band for which there exists, in the same age band, a raw score that is lower that yields the same scaled score. Norm table entries that were classified as floor- or ceiling-effects were removed from the dataset.

\section{Methods}

In order to gain insight into the relationship between scaled scores across age for a particular raw score, the data was first visualized. For each combination of test, subtest and raw score, the scaled score was plotted against age. Some examples of these plots can be seen in figures 1 and 2 .

Figure 1(a) depicts the scaled scores belonging to a raw score of 11 on the categories subtest for the SON-R 2-8. A 6-year-old child that correctly answers 11 items on this subtest receives an average scaled score $(z=0)$. For increasingly younger children, this same raw score equals increasingly higher scaled scores. For the youngest ages a ceiling is visible. The youngest children should probably receive a scaled score higher than 3 standard deviations above the mean, but the maximum scaled score in the norm table of this test (and many other tests) was 3 standard deviations above the mean.

In figure 2(b), quite a large floor effect is clearly visible. Children above the age of 10 who attain a raw score of 8 on this subtest should receive a scaled score lower than 3 SD below the mean.

The scaled scores that show a floor- or ceiling effect were removed from the dataset, but are shown here for illustrative purposes. 


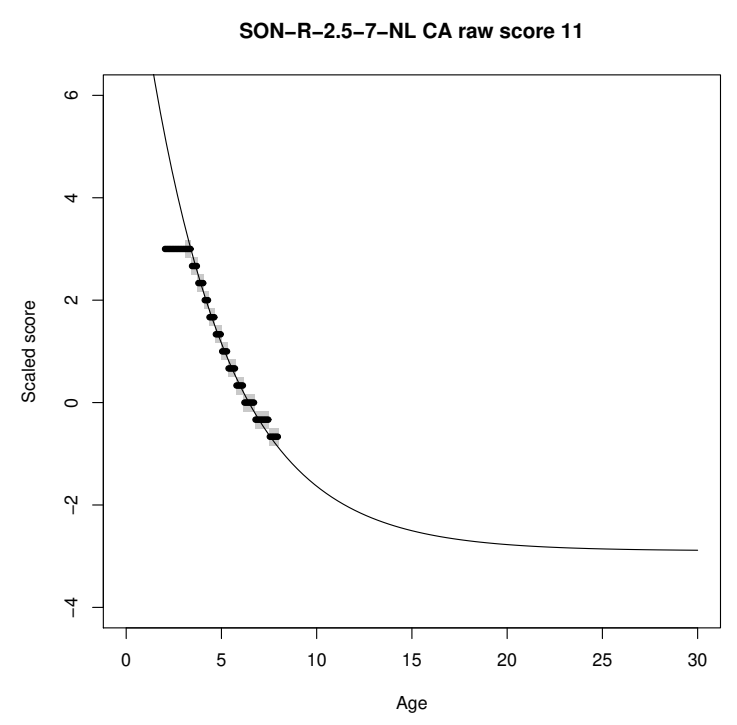

(a)

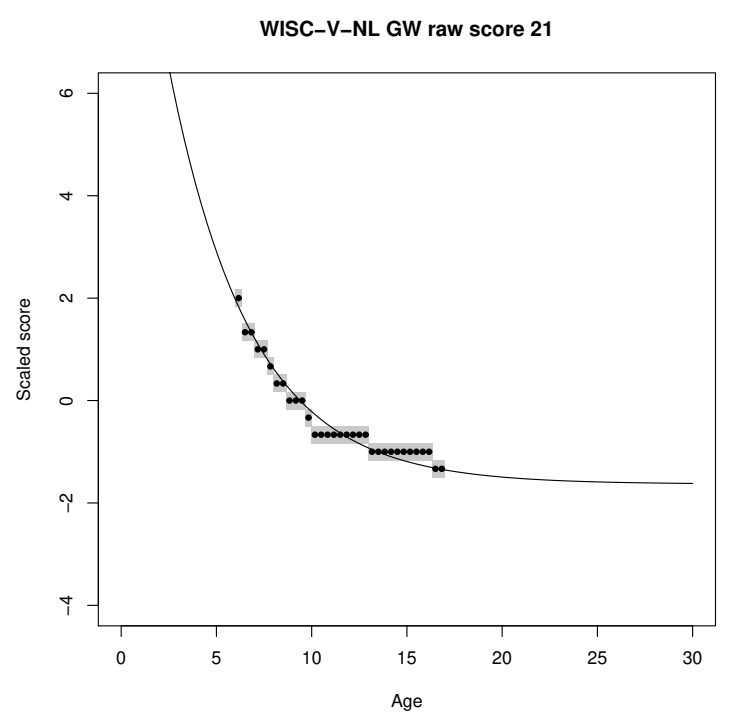

(c)

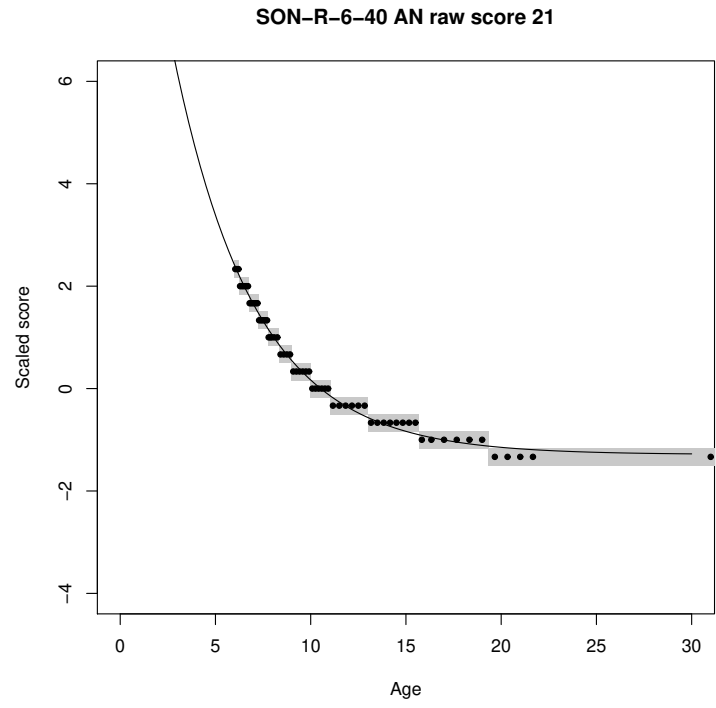

(b)

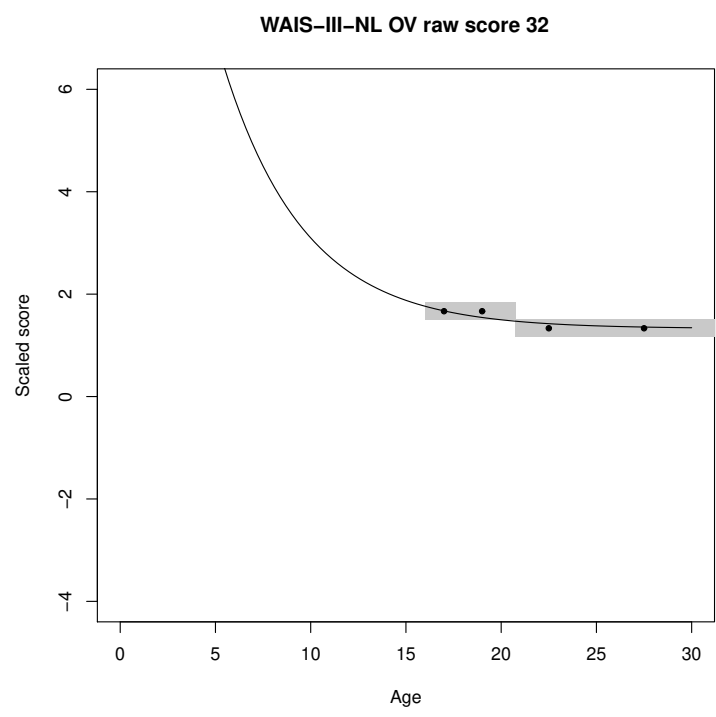

(d)

Figure 1: Scaled scores plotted against age, for four different raw scores in 4 different subtests (from four different tests). The grey bars indicate possible rounding effects in the norm tables, points without grey bars indicate removed entries due to floor- or ceiling-effects. The black line indicates the fitted function 1 


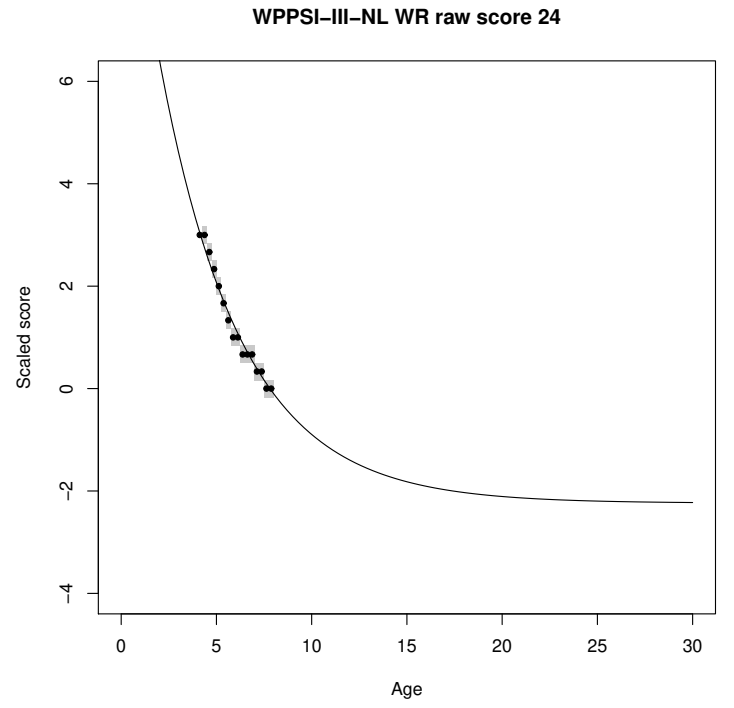

(a)

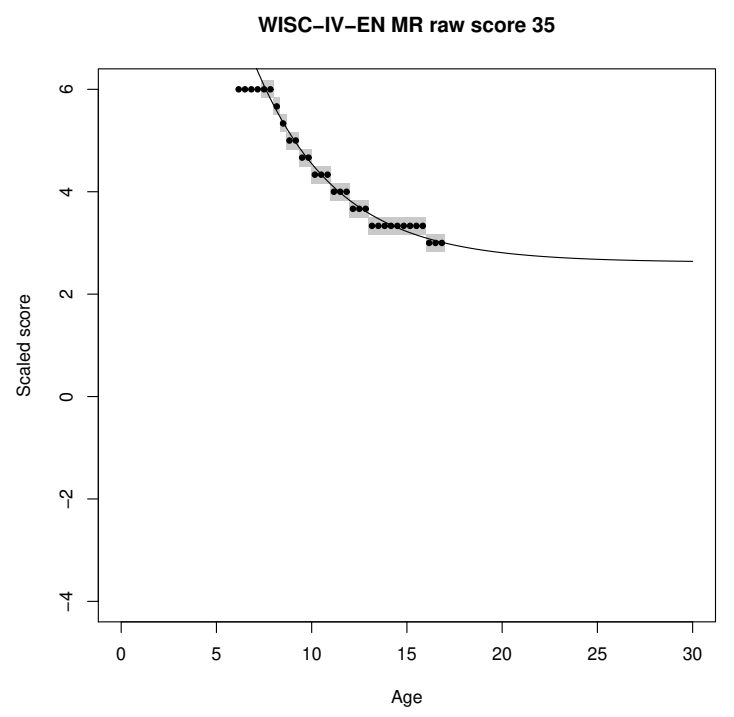

(c)

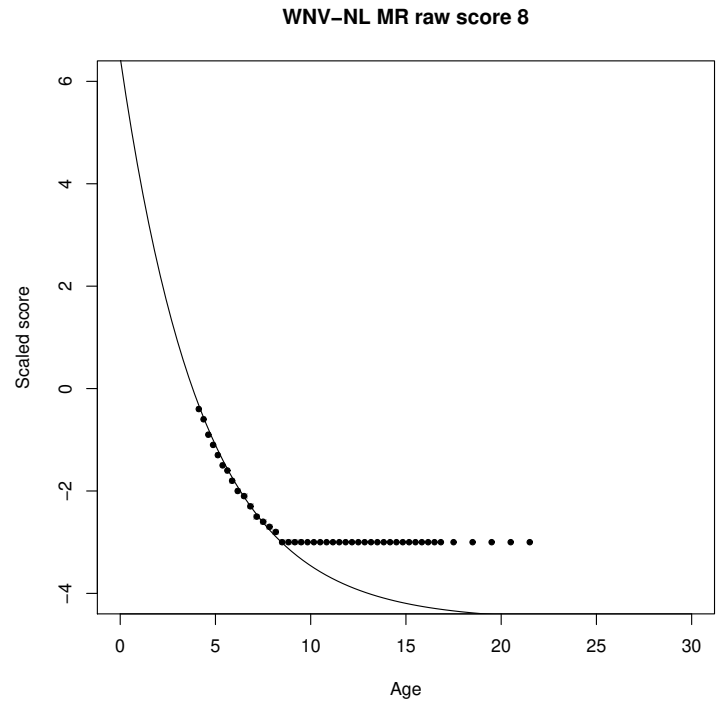

(b)

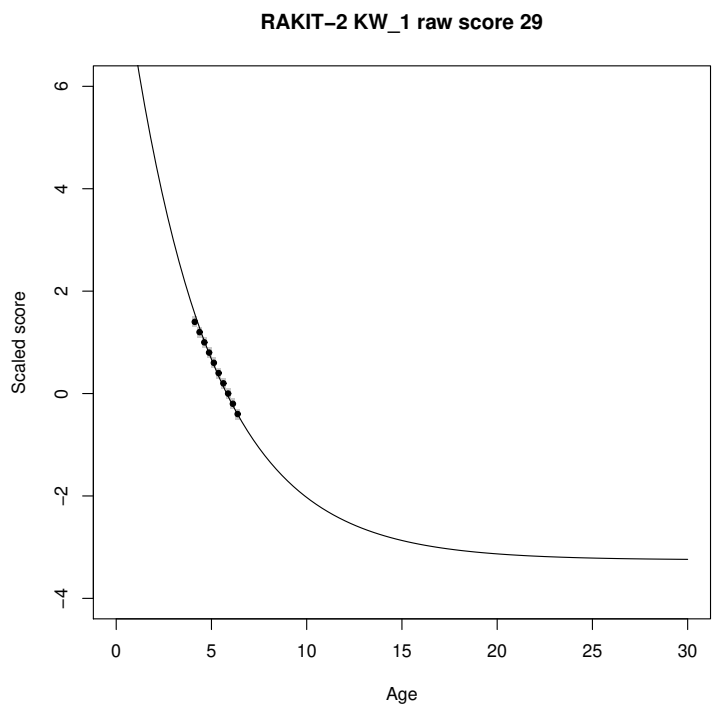

(d)

Figure 2: Scaled scores plotted against age, for four different raw scores in 4 different subtests (from four different tests). The grey bars indicate possible rounding effects in the norm tables, points without grey bars indicate removed entries due to floor- or ceiling-effects. In (b) and (d) the error bars are too small to be visible. The black line indicates the fitted function 1

When looking at these plots, a common pattern becomes visible. Performance that receives an average scaled score $(z=0)$ for adults yields increasingly higher scaled scores for younger ages. In a way 
this is very intuitive, children that are able to perform a mental task equally well as an average adult are most likely to be of above-average intelligence. Performance that is given an average scaled score in childhood equates to below average scaled scores for adults. Again this is intuitive, adults that perform mental tasks equally well as the average 10-year old child are likely to be of below-average intelligence. In general, the scaled scores for any raw score, when plotted against age, show a striking similarity: a steep descent before age 10 which gradually stabilizes into almost no change after age 20.

This general pattern, which appeared for almost all raw scores in each subtest, led to the belief that for any raw score in any subtest the scaled score could be modeled as a continuous function of age. The steep descent in younger ages, followed by a gradual tapering off in adulthood led to the belief that the function should contain a horizontal asymptote. This thus meant that the function needed to be in the form of a fraction where the variable age appeared in the denominator.

After experimenting with several types of functions, the data was modeled as:

$$
\begin{array}{r}
f: \mathbb{R}^{+} \times \mathbb{R} \rightarrow \mathbb{R} \\
s=f(x, p)=\left(\frac{p+c_{3}}{e^{c_{1}+c_{2} x}}\right)+p
\end{array}
$$

where $s$ is the scaled score, $x$ the age, $\left\{c_{1}, c_{2}, c_{3}\right\}$ the coefficients to be estimated and $p$ a parameter to scale the function to a specific raw score (the 'difficulty' parameter).

Coefficients $\left\{c_{1}, c_{2}, c_{3}\right\}$ were estimated used least squares regression in the following way: for a given candidate set of coefficients $\left(\left\{c_{1}, c_{2}, c_{3}\right\}\right)$, the penalty was defined as follows: for each raw score set (a set of $n$ data points belonging to a combination of a test, a subtest, and a raw score), the difficulty parameter $p$ was estimated by minimizing the function:

$$
\sum_{i=1}^{n}\left(f\left(x_{i}, p\right)-s_{i}\right)^{2}
$$

where $x_{i}$ is the age and $s_{i}$ the scaled score belonging to data point $i$.

With the parameter $p$ then estimated for this score set, the error for each data point $i$ in this score set was then defined as the squared difference between $f\left(x_{i}, p\right)$ and $s_{i}$.

With the error for each data point calculated, the penalty for this set of candidate coefficients $\left\{c_{1}, c_{2}, c_{3}\right\}$ was then defined as the weighted sum of the error of all data points, with the weights defined in a way so that each test would be weighted the same, regardless of the number of data points associated with the test. For example, if there are $m$ data points belonging to test $k$, the weight of any data point belonging tot test $k$ would be $\frac{1}{m}$.

The coefficients $\left\{c_{1}, c_{2}, c_{3}\right\}$ were then estimated by minimizing the penalty function by using a quasi-Newton method (also known as a variable metric algorithm by Broyden, Fletcher, Goldfarb and Shanno). 


\section{Results}

In order to measure the fit of the model, the error was calculated for each data point. For each data point $i$, the error is defined as the difference between the scaled score predicted by the DARSIS model, and the actual scaled score $\left(\hat{s_{i}}-s_{i}\right)$. The Root Mean Square Error (RMSE) for the different subtests is then defined as the standard deviation of the errors for all data points that belong to a certain subtest. The RMSE per subtest is shown in table 1, along with the amount of variance explained and several other measures.

Note that usually when norm tables are created, scaled scores are rounded to the nearest integer, thus their maximum deviation from their 'true' value is 0.5. To indicate the expected amount of error in the model due to rounding errors in the norm table, the expected Root Mean Square Error (eRMSE) is calculated. For any norm table, the eRMSE is given by:

$$
\mathrm{eRMSE}=\frac{\sigma_{a}}{\sigma_{b}}=\frac{\frac{\sqrt{3}}{6}}{\sigma_{b}} \approx \frac{0.29}{\sigma_{b}}
$$

where $\sigma_{a}=\frac{\sqrt{3}}{6}$ is the standard deviation of the standard uniform distribution $(U(0,1))$, and $\sigma_{b}$ is the standard deviation used to create the norm table. For example, a 'Wechsler-style' norm table, that uses a mean of 10 and a standard deviation of 3 , has an eRMSE of $\approx 0.096$. T-scores, with a standard deviation of 10 , have an eRMSE of $\approx 0.029$. The corrected Root Mean Square Error (cRMSE) is the RMSE minus the eRMSE and gives an indication of the true RMSE, without the rounding in the norm tables. 


\begin{tabular}{|c|c|c|c|c|c|}
\hline Test & Subtest & RMSE & eRMSE & cRMSE & Variance explained \\
\hline RAKIT-2 & EX_2 & 0.066 & 0.058 & 0.008 & 99.837 \\
\hline RAKIT-2 & EX_1 & 0.067 & 0.058 & 0.009 & 99.851 \\
\hline RAKIT-2 & EX_3 & 0.068 & 0.058 & 0.010 & 99.801 \\
\hline RAKIT-2 & AN_3 & 0.074 & 0.058 & 0.016 & 99.687 \\
\hline WAIS-III-NL & MR & 0.098 & 0.096 & 0.002 & 99.519 \\
\hline WAIS-IV-NL & GW & 0.106 & 0.096 & 0.010 & 99.591 \\
\hline WAIS-IV-NL & FS & 0.107 & 0.096 & 0.011 & 99.555 \\
\hline WNV-NL & RO & 0.108 & 0.029 & 0.079 & 99.676 \\
\hline RAKIT-2 & AN_2 & 0.120 & 0.058 & 0.062 & 99.296 \\
\hline RAKIT & EX_1 & 0.123 & 0.058 & 0.066 & 99.460 \\
\hline RAKIT-2 & KW_3 & 0.124 & 0.058 & 0.067 & 99.095 \\
\hline RAKIT & EX_3 & 0.125 & 0.058 & 0.067 & 99.382 \\
\hline GOS-2.5-4.5 & MS & 0.126 & 0.010 & 0.116 & 99.520 \\
\hline COVAT-CHC & FR & 0.126 & 0.096 & 0.030 & 99.243 \\
\hline WAIS-III-NL & OV & 0.131 & 0.096 & 0.035 & 99.055 \\
\hline KAIT-NL & $\mathrm{SL}$ & 0.132 & 0.096 & 0.036 & 98.804 \\
\hline RAKIT & $\mathrm{KW} \_3$ & 0.133 & 0.058 & 0.076 & 99.263 \\
\hline KAIT-NL & LR & 0.134 & 0.096 & 0.037 & 99.186 \\
\hline COVAT-CHC & $\mathrm{DP}$ & 0.135 & 0.096 & 0.039 & 99.011 \\
\hline COVAT-CHC & $\mathrm{PR}$ & 0.138 & 0.096 & 0.041 & 99.330 \\
\hline RAKIT & AN_2 & 0.141 & 0.058 & 0.083 & 99.045 \\
\hline KAIT-NL & $\mathrm{GC}$ & 0.144 & 0.096 & 0.048 & 99.233 \\
\hline WPPSI-III-NL & MR & 0.147 & 0.096 & 0.051 & 99.291 \\
\hline WAIS-IV-NL & MR & 0.152 & 0.096 & 0.056 & 99.049 \\
\hline WPPSI-IV-NL & $\mathrm{PC}$ & 0.154 & 0.096 & 0.058 & 99.133 \\
\hline RAKIT & EX_2 & 0.154 & 0.058 & 0.097 & 99.247 \\
\hline WPPSI-III-NL & $\mathrm{PC}$ & 0.159 & 0.096 & 0.063 & 99.180 \\
\hline WPPSI-III-NL & WR & 0.162 & 0.096 & 0.066 & 98.889 \\
\hline RAKIT & AN_3 & 0.168 & 0.058 & 0.110 & 98.759 \\
\hline WPPSI-IV-NL & $\mathrm{MR}$ & 0.173 & 0.096 & 0.076 & 98.892 \\
\hline WISC-IV-EN & $\mathrm{MR}$ & 0.179 & 0.096 & 0.083 & 97.876 \\
\hline RAKIT-2 & $\mathrm{KW}_{-} 2$ & 0.184 & 0.058 & 0.126 & 98.471 \\
\hline WISC-V-EN & $\mathrm{PC}$ & 0.185 & 0.096 & 0.089 & 97.065 \\
\hline COVAT-CHC & $\mathrm{TE}$ & 0.192 & 0.096 & 0.095 & 98.786 \\
\hline WISC-R-NL & $\mathrm{BP}$ & 0.196 & 0.096 & 0.100 & 98.291 \\
\hline WISC-V-NL & $\mathrm{MR}$ & 0.197 & 0.096 & 0.101 & 98.899 \\
\hline RAVEN & SPM & 0.200 & 0.038 & 0.161 & 97.909 \\
\hline WPPSI-IV-NL & OV & 0.204 & 0.096 & 0.107 & 98.545 \\
\hline GOS-2.5-4.5 & WS & 0.204 & 0.010 & 0.194 & 98.775 \\
\hline WISC-IV-EN & WR & 0.206 & 0.096 & 0.110 & 95.891 \\
\hline RAKIT & KW_2 & 0.207 & 0.058 & 0.149 & 98.210 \\
\hline RAKIT & KW_1 & 0.236 & 0.058 & 0.178 & 97.709 \\
\hline WISC-V-NL & OV & 0.244 & 0.096 & 0.148 & 98.274 \\
\hline WISC-R-NL & OV & 0.253 & 0.096 & 0.157 & 98.039 \\
\hline WAIS-IV-NL & OV & 0.261 & 0.096 & 0.165 & 97.601 \\
\hline WISC-V-EN & MR & 0.262 & 0.096 & 0.166 & 93.883 \\
\hline WNV-NL & MR & 0.264 & 0.029 & 0.235 & 98.324 \\
\hline WISC-V-NL & GW & 0.264 & 0.096 & 0.168 & 97.960 \\
\hline WISC-V-EN & VP & 0.265 & 0.096 & 0.169 & 91.499 \\
\hline WNV-NL & FL & 0.272 & 0.029 & 0.243 & 97.591 \\
\hline RAKIT-2 & KW_1 & 0.274 & 0.058 & 0.216 & 97.398 \\
\hline SON-R-2.5-7-NL-DE & $\mathrm{CA}$ & 0.275 & 0.096 & 0.179 & 98.166 \\
\hline SON-R-2.5-7-NL-DE & AN & 0.284 & 0.096 & 0.188 & 97.581 \\
\hline SON-R-6-40 & $\mathrm{CA}$ & 0.289 & 0.096 & 0.192 & 97.724 \\
\hline SON-R-6-40 & AN & 0.319 & 0.096 & 0.223 & 97.041 \\
\hline SON-R-2.5-7-NL & AN & 0.320 & 0.096 & 0.223 & 97.255 \\
\hline SON-R-2.5-7-NL & $\mathrm{CA}$ & 0.321 & 0.096 & 0.225 & 97.213 \\
\hline WISC-III-NL & $\mathrm{RE}$ & 0.365 & 0.096 & 0.269 & 96.247 \\
\hline WISC-IV-EN & $\mathrm{PC}$ & 0.372 & 0.096 & 0.275 & 90.258 \\
\hline WISC-III-NL & OV & 0.384 & 0.096 & 0.287 & 95.908 \\
\hline
\end{tabular}

Table 1: RMSE, expected RMSE, corrected RMSE and variance explained for each subtest

As can be seen in table 1, the DARSIS model explains a very large portion of the variance in the subtest norms. The RMSE ranges from around 0.07 to 0.38 standard deviations. The corrected RMSE is between 5-98\% smaller than the uncorrected RMSE, with a mean of $48 \%$. This indicates that a substantial portion of the error is due to the rounding of the scaled scores in the norm tables.

\subsection{Crossvalidation}

Usually in regression and prediction statistics some sort of crossvalidation is performed to prevent the prediction statistics from being too optimistic. Since norm tables themselves are often the result of some kind of regression procedure, randomly removing some entries from a norm table means that no information is really lost, these data points could easily be reconstructed. Therefore a Leave One Out Crossvalidation (LOOC) was done, where in each instance one of the tests was excluded from 
the training set and used as the test set. For example, the prediction statistics for the subtests of the WISC-V were determined by first estimating the model coefficients $\left\{c_{1}, c_{2}, c_{3}\right\}$ on all data except the WISC-V (the training set), and then validating the model with the data from the WISC-V (the test set).

\begin{tabular}{|c|c|c|c|c|c|}
\hline Test & Subtest & RMSE & eRMSE & cRMSE & Variance explained \\
\hline RAKIT-2 & EX_2 & 0.073 & 0.058 & 0.015 & 99.898 \\
\hline RAKIT-2 & EX_3 & 0.075 & 0.058 & 0.017 & 99.880 \\
\hline RAKIT-2 & EX_1 & 0.078 & 0.058 & 0.020 & 99.897 \\
\hline RAKIT-2 & AN_3 & 0.081 & 0.058 & 0.023 & 99.812 \\
\hline WNV-NL & RO & 0.121 & 0.029 & 0.092 & 99.799 \\
\hline WAIS-III-NL & MR & 0.123 & 0.096 & 0.027 & 99.622 \\
\hline WAIS-IV-NL & GW & 0.131 & 0.096 & 0.035 & 99.687 \\
\hline RAKIT-2 & AN_2 & 0.132 & 0.058 & 0.075 & 99.572 \\
\hline WAIS-IV-NL & FS & 0.136 & 0.096 & 0.040 & 99.643 \\
\hline RAKIT-2 & KW_3 & 0.137 & $\begin{array}{l}0.058 \\
0.058\end{array}$ & $\begin{array}{l}0.079 \\
0.079\end{array}$ & 99.453 \\
\hline GOS-2.5-4.5 & $\mathrm{MS}$ & 0.139 & 0.010 & 0.129 & 99.707 \\
\hline RAKIT & EX_3 & 0.143 & 0.058 & 0.085 & 99.592 \\
\hline COVAT-CHC & FR & 0.144 & 0.096 & 0.048 & 99.503 \\
\hline RAKIT & EX_1 & 0.149 & 0.058 & 0.091 & 99.607 \\
\hline COVAT-CHC & $\mathrm{DP}$ & 0.154 & 0.096 & 0.058 & 99.356 \\
\hline COVAT-CHC & PR & 0.155 & 0.096 & 0.059 & 99.574 \\
\hline $\begin{array}{l}\text { RAKIT } \\
\text { RAT }\end{array}$ & KW_3 & 0.155 & 0.058 & 0.097 & 99.500 \\
\hline KAIT-NL & LR & 0.157 & 0.096 & 0.061 & 99.435 \\
\hline KAIT-NL & $\mathrm{SL}$ & 0.159 & 0.096 & 0.063 & 99.135 \\
\hline WPPSI-III-NL & MR & 0.163 & 0.096 & 0.066 & 99.570 \\
\hline WAIS-III-NL & OV & 0.165 & $\begin{array}{l}0.096 \\
0.096\end{array}$ & $\begin{array}{l}0.060 \\
0.069\end{array}$ & 99.232 \\
\hline KAIT-NL & $\mathrm{GC}$ & 0.169 & 0.096 & 0.073 & 99.469 \\
\hline RAKIT & AN_2 & 0.169 & 0.058 & 0.112 & 99.311 \\
\hline WPPSI-IV-NL & $\mathrm{PC}$ & 0.172 & 0.096 & 0.076 & 99.450 \\
\hline WPPSI-III-NL & WR & 0.173 & 0.096 & 0.077 & 99.358 \\
\hline WPPSI-III-NL & $\mathrm{PC}$ & 0.176 & 0.096 & 0.080 & 99.504 \\
\hline RAKIT & EX_2 & 0.186 & 0.058 & 0.128 & 99.453 \\
\hline WISC-IV-EN & $\mathrm{MR}$ & 0.187 & 0.096 & 0.091 & 98.713 \\
\hline RAKIT & AN_3 & 0.189 & 0.058 & 0.131 & 99.207 \\
\hline WAIS-IV-NL & $\mathrm{MR}$ & 0.196 & 0.096 & 0.100 & 99.194 \\
\hline WPPSI-IV-NL & $\mathrm{MR}$ & 0.197 & 0.096 & 0.100 & 99.270 \\
\hline RAKIT-2 & KW_2 & 0.203 & $\begin{array}{l}0.058 \\
0.058\end{array}$ & 0.145 & 99.073 \\
\hline WISC-V-EN & $\mathrm{PC}$ & 0.205 & 0.096 & 0.108 & 98.262 \\
\hline WISC-R-NL & $\mathrm{BP}$ & 0.213 & 0.096 & 0.117 & 98.996 \\
\hline WISC-V-NL & MR & 0.213 & 0.096 & 0.117 & 99.359 \\
\hline GOS-2.5-4.5 & WS & 0.218 & 0.010 & 0.208 & 99.300 \\
\hline COVAT-CHC & $\mathrm{TE}$ & 0.219 & 0.096 & 0.123 & 99.194 \\
\hline RAVEN & SPM & 0.227 & 0.038 & 0.189 & 98.618 \\
\hline WISC-IV-EN & WR & 0.228 & 0.096 & 0.131 & 97.673 \\
\hline WPPSI-IV-NL & OV & 0.228 & 0.096 & 0.132 & 99.086 \\
\hline WISC-V-NL & OV & 0.247 & 0.096 & 0.151 & 99.103 \\
\hline RAKIT & KW_2 & 0.253 & 0.058 & 0.195 & 98.641 \\
\hline WISC-R-NL & $\mathrm{OV}^{-2}$ & 0.277 & 0.096 & 0.181 & 98.813 \\
\hline SON-R-2.5-7-NL-DE & AN & 0.281 & 0.096 & 0.185 & 98.771 \\
\hline $\begin{array}{l}\text { RAKIT } \\
\text { RKT }\end{array}$ & KW_1 & 0.284 & 0.058 & 0.226 & 98.332 \\
\hline WISC-V-NL & GW & 0.285 & 0.096 & 0.189 & 98.818 \\
\hline WISC-V-EN & VP & 0.286 & 0.096 & 0.190 & 94.932 \\
\hline WNV-NL & MR & 0.288 & $\begin{array}{l}0.029 \\
0.029\end{array}$ & 0.260 & 99.026 \\
\hline WISC-V-EN & $\mathrm{MR}$ & 0.288 & 0.096 & 0.192 & 96.201 \\
\hline WNV-NL & $\mathrm{FL}$ & 0.305 & 0.029 & 0.276 & 98.500 \\
\hline SON-R-2.5-7-NL-DE & $\mathrm{CA}$ & 0.308 & 0.096 & 0.212 & 98.871 \\
\hline RAKIT- 2 & KW_1 & 0.308 & 0.058 & 0.251 & 98.327 \\
\hline SON-R-2.5-7-NL & AN & 0.322 & 0.096 & 0.226 & 98.572 \\
\hline WAIS-IV-NL & OV & 0.323 & 0.096 & 0.227 & 98.114 \\
\hline SON-R-6-40 & $\mathrm{CA}$ & 0.343 & 0.096 & 0.247 & 98.451 \\
\hline SON-R-2.5-7-NL & $\mathrm{CA}$ & 0.345 & 0.096 & 0.249 & 98.374 \\
\hline SON-R-6-40 & AN & 0.372 & 0.096 & 0.276 & 98.062 \\
\hline WISC-IV-EN & $\mathrm{PC}$ & 0.400 & 0.096 & 0.304 & 94.706 \\
\hline WISC-III-NL & $\mathrm{RE}$ & 0.411 & 0.096 & 0.315 & 97.565 \\
\hline WISC-III-NL & OV & 0.432 & 0.096 & 0.336 & 97.321 \\
\hline
\end{tabular}

Table 2: RMSE, expected RMSE, corrected RMSE and variance explained for each subtest in the LOOC.

As can be seen in table 2 , the results are similar to the results in section 4 . The percentage of explained variance in the norm tables is still very high, with most norm tables having almost all their variance explained by the model. The RMSE ranges from around 0.08 to 0.43 standard deviations. The corrected RMSE is between 4-79\% smaller than the uncorrected RMSE, with a mean of $42 \%$. This indicates that also in the LOOC, a substantial portion of the error is due to the rounding of the scaled scores in the norm tables. 


\subsection{Error Analysis}

It was expected that some norms table entries would be more accurately predicted by the DARSIS model. The first variable for which a difference in the accuracy of prediction was expected was the variable $s$, the scaled score. Scaled scores around the mean $(s=0)$ are usually the easiest to estimate, which results in norm tables being the most accurate around this 50-th percentile. Figure 3 shows the RMSE for data points grouped by the absolute value of $s$ (rounded off to the nearest 0.1 to facilitate the creation of the groups). Because it was expected that scaled scores around $s=$ 0 would be estimated most accurately, with larger errors for scaled scores that deviate more from the mean, the absolute value of $s$ is used in the x-axis. Scaled scores more than 3 SD away from the mean are not included in this error analysis, since only very few tests report norms for these extremes.

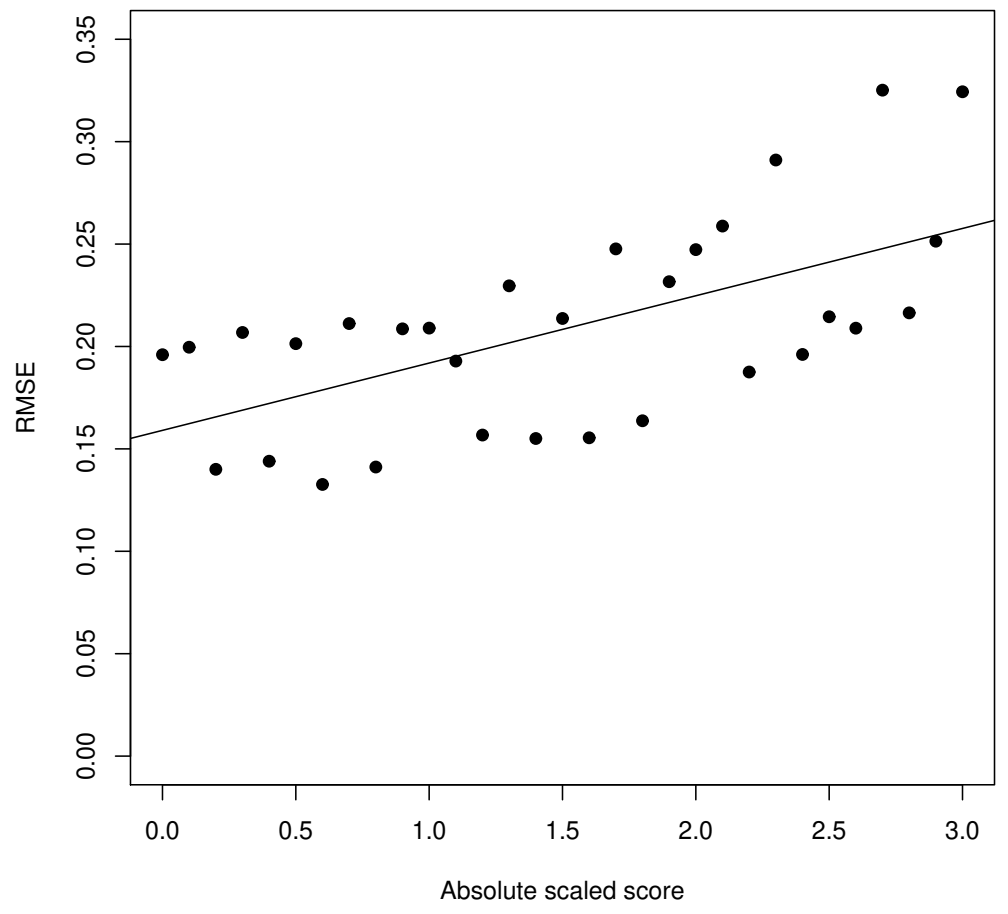

Figure 3: The RSME for different values of absolute $s$

The line shows a simple linear regression to predict the RMSE based on the absolute value of $s$. A significant regression equation was found $(F(1,29)=16.801, p<.001)$, with an $R^{2}$ of 0.367 . RMSE increased with 0.03 points on the $z$-scale for each unit of deviation of $s$ from the mean. This indicated that norm table entries corresponding to a mean scaled score $(s=0)$ are estimated the most accurately by the DARSIS model.

A second variable of interest that might influence the accuracy of the DARSIS model is the variable 
$p$. Note that $p$ is a 'difficulty parameter', where higher values of $p$ indicate better performance and lower values of $p$ indicate worse performance. Figure 4 shows the RMSE for data points grouped by their value of $p$ (rounded to the nearest 0.1 ).

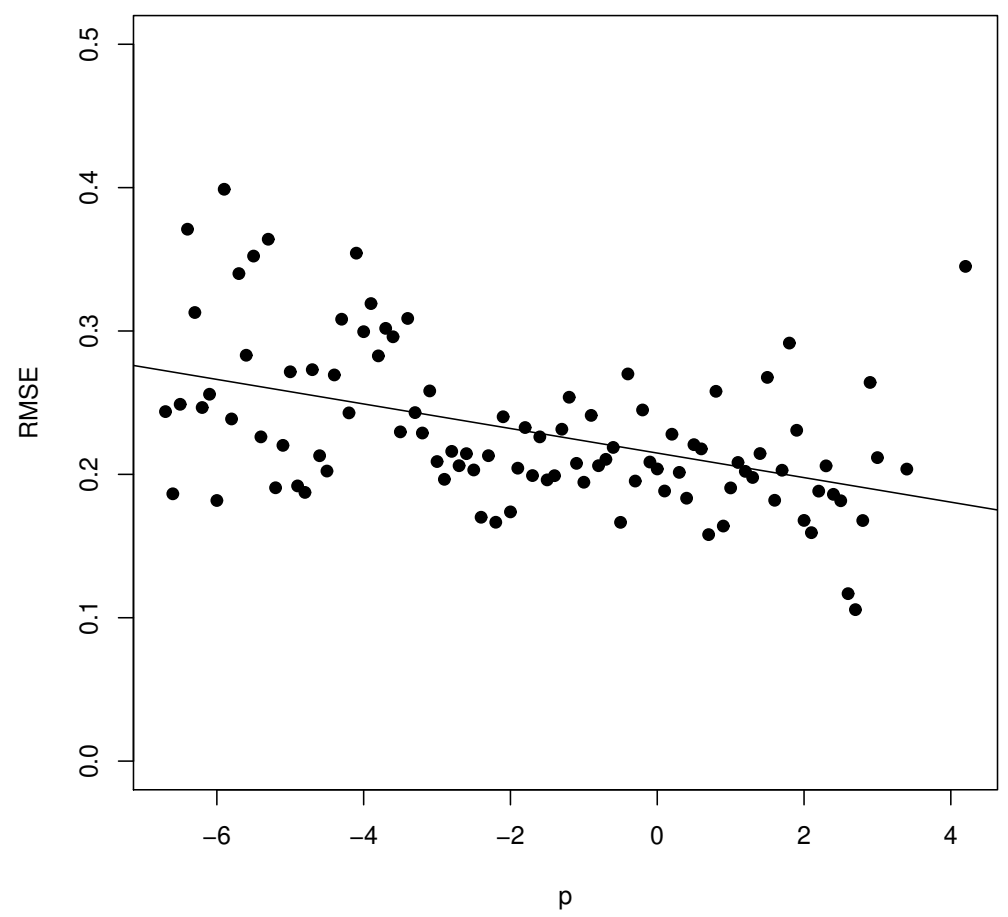

Figure 4: The RSME for different values of $p$

As can be seen in figure 4, higher values of $p$ result in a smaller RMSE from the DARSIS model. The line shows a simple linear regression to predict the RMSE based on the value of $p$. A significant regression equation was found $(F(1,98)=26.177, p<.000)$, with an $R^{2}$ of 0.211 . RMSE decreased with 0.0086 points on the $z$-scale for each unit increase in $p$. Although age could be considered as a confounding variable here, a similar analysis of the RMSE with regards to age did not reach statistical significance $(F(1,148)=3.4879, p=.064)$.

Another variable for which statistical significance was not reached was the year in which a test was normed $(F(1,13)=0.0451, p=0.835)$. This is a first indication that the DARSIS model is relatively stable across time, at least for the time period 1984-2020. 


\section{Discussion}

With the estimation of the coefficients $\left\{c_{1}, c_{2}, c_{3}\right\}$, the general pattern of equal mental performance across age becomes clear. In figure 5 , we see function $f$ plotted for several values of $p$. In figures 1 and 2 , the black lines represent $f(x, p)$ with the estimated coefficients.

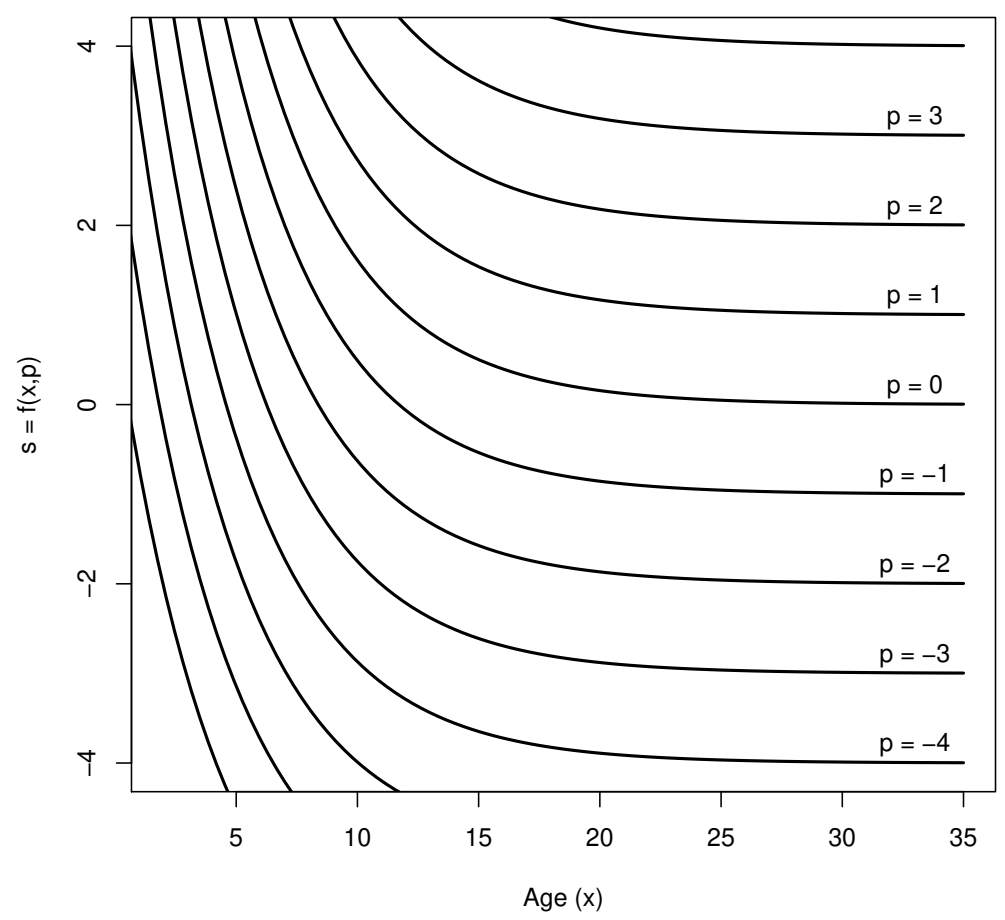

Figure 5: $f(x, p)$ (function 1) for several values of $p$

Since we have that $s=f(x, p)$ (function 1 ), we also have that:

$$
p=p(x, s)=\frac{s e^{c_{1}+c_{2} x}-c_{3}}{e^{c_{1}+c_{2} x}+1} \quad\left(e^{c_{1}+c_{2} x} \neq 0\right)
$$

which gives us figure 6 , which shows the value of $p$ (the 'difficulty' parameter) against age for several values of $s$. Of course, figure 6 can also be seen as percentile curves, since $s=0$ equals the 50-th percentile, $s=1$ the 84 -th percentile, etc. Again figure 6 shows how small children develop very rapidly, the growth of intellectual development slows down at the start of puberty, and almost no change occurs after age 20 . 


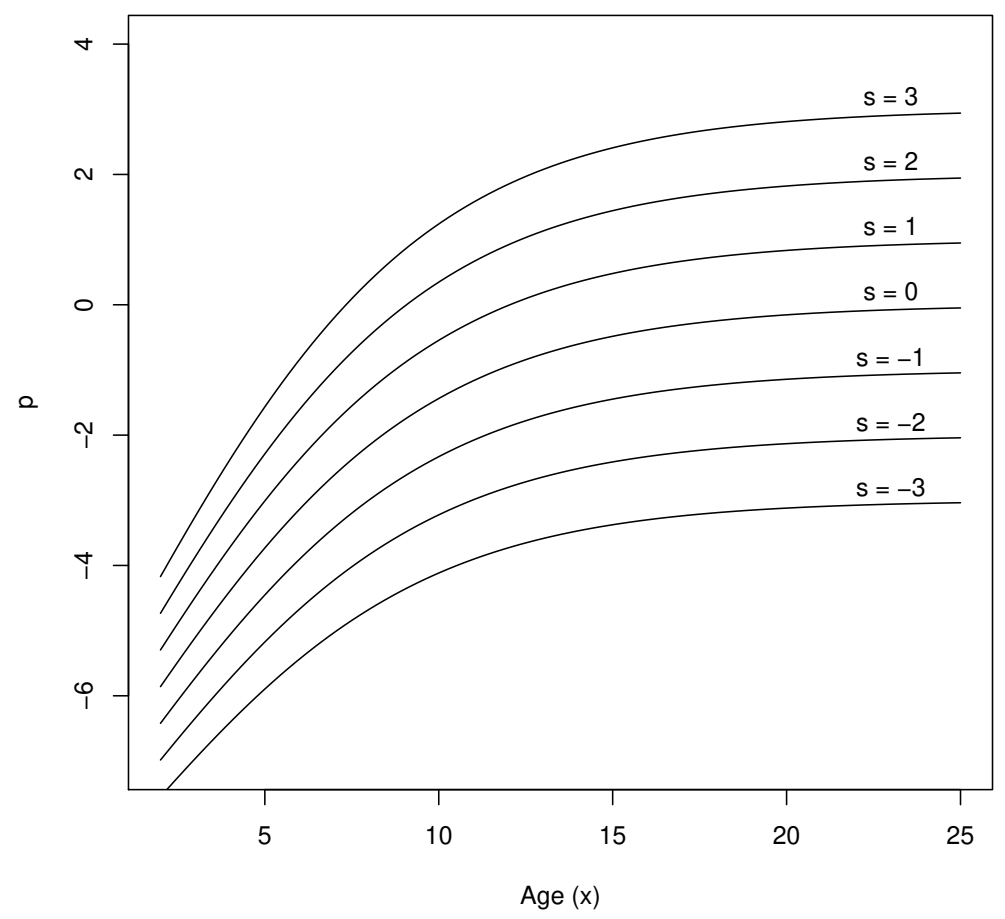

Figure 6: values of $p$ plotted against age, for several values of $s$, using function 4

\subsection{Limitations}

Although the DARSIS model fits the data very well, there are several limitations. Firstly, data was only available from a minimum age of 2 and onward. Although the DARSIS model could theoretically be extended down to age 0 , it is not known if the model would still reflects the underlying changes in score distributions for these very young children. Secondly, norm tables were only included up to the point where the raw score associated with average performance started to show a declining trend. The general cognitive decline associated with older age is thus not modeled by the DARSIS model, although a similar formula could be used in future research to do so. This also means that it is unknown up to which age this version of the DARSIS model still applies, although a maximum age of around 20-25 seems reasonable. Lastly, further research should be undertaken to validate the DARSIS model using different norm tables, preferably from different countries and different years of standardization. 


\section{Applications}

\subsection{Above-level (or below-level) testing}

Above-level testing (sometimes also called out-of-level testing) is the practice of administering a test to a child that is intended and normed for children of a higher age or grade. Above-level testing is often used to reduce ceiling effects that are present in age-appropriate tests and/or to increase score variability in order to better discriminate between moderately and highly gifted children. For a comprehensive review about above-level testing, see (Warne, 2012).

Using the DARSIS model, test norms can be extrapolated to create norms for younger (or older) children outside the ages for which the test was intended and normed. Although there will always be some error in these norm tables, the amount of error in these norms would be similar to the amount of error found in the LOOC in section 4.1, which can be considered relatively small.

In table 3 an example is given of an extrapolated test norm. The norm in question is the third age band of the exclusion subtest from the RAKIT-2. In the original norm table, ages are given in 3 -month intervals starting at age 9.5. Using only the norm table data from age 10 and older and the DARSIS model, scaled scores were estimated for the age of 9 years and 6 months to 9 years and 8 months. Since the original norm table also started at age 9 years and 6 months, the extrapolated norm table can be compared to the original norm table. The predicted norm, compared to the real norm, deviates by a maximum of 1.2 scaled score points, which translates to 0.24 standard deviations (or 3.6 IQ points using a SD of 15). The RMSE is 0.075 standard deviations (equal to 1.12 IQ points). Since the measurement error of a very reliable test, with a reliability of 0.95 , would be around 0.22 standard deviations (equal to $3.35 \mathrm{IQ}$ points), the error in the predicted norm is considerably less than the measurement error of the test itself. 


\begin{tabular}{|c|c|c|c|c|c|c|c|c|c|}
\hline Raw score & 9.625 (predicted) & 9.625 (real) & 10.625 & 10.875 & 11.125 & 11.375 & 11.625 & 11.875 & 12.125 \\
\hline 17 & & 0 & & & & & & & \\
\hline 18 & 1.3 & 1 & 0 & & & & & & \\
\hline 19 & 2 & 2 & 1 & 0 & 0 & 0 & & & \\
\hline 20 & 2.7 & 2 & 1 & 1 & 1 & 1 & 0 & 0 & 0 \\
\hline 21 & 3.4 & 3 & 2 & 2 & 1 & 1 & 1 & 1 & 1 \\
\hline 22 & 3.8 & 3 & 2 & 2 & 2 & 2 & 1 & 1 & 1 \\
\hline 23 & 4.4 & 4 & 3 & 3 & 2 & 2 & 2 & 2 & 2 \\
\hline 24 & 4.8 & 4 & 3 & 3 & 3 & 3 & 2 & 2 & 2 \\
\hline 25 & 5.2 & 5 & 4 & 3 & 3 & 3 & 3 & 3 & 2 \\
\hline 26 & 5.7 & 5 & 4 & 4 & 4 & 3 & 3 & 3 & 3 \\
\hline 27 & 6.1 & 6 & 5 & 4 & 4 & 4 & 4 & 3 & 3 \\
\hline 28 & 6.5 & 6 & 5 & 5 & 4 & 4 & 4 & 4 & 4 \\
\hline 29 & 6.9 & 7 & 5 & 5 & 5 & 5 & 4 & 4 & 4 \\
\hline 30 & 7.3 & 7 & 6 & 5 & 5 & 5 & 5 & 5 & 4 \\
\hline 31 & 7.8 & 7 & 6 & 6 & 6 & 5 & 5 & 5 & 5 \\
\hline 32 & 7.9 & 8 & 6 & 6 & 6 & 6 & 5 & 5 & 5 \\
\hline 33 & 8.4 & 8 & 7 & 7 & 6 & 6 & 6 & 6 & 5 \\
\hline 34 & 8.8 & 9 & 7 & 7 & 7 & 6 & 6 & 6 & 6 \\
\hline 35 & 9.2 & 9 & 8 & 7 & 7 & 7 & 7 & 6 & 6 \\
\hline 36 & 9.5 & 9 & 8 & 8 & 7 & 7 & 7 & 7 & 6 \\
\hline 37 & 9.9 & 10 & 8 & 8 & 8 & 7 & 7 & 7 & 7 \\
\hline 38 & 10.3 & 10 & 9 & 8 & 8 & 8 & 8 & 7 & 7 \\
\hline 39 & 10.8 & 11 & 9 & 9 & 9 & 8 & 8 & 8 & 8 \\
\hline 40 & 11.2 & 11 & 10 & 9 & 9 & 9 & 8 & 8 & 8 \\
\hline 41 & 11.7 & 11 & 10 & 10 & 10 & 9 & 9 & 9 & 8 \\
\hline 42 & 12 & 12 & 10 & 10 & 10 & 9 & 9 & 9 & 9 \\
\hline 43 & 12.4 & 12 & 11 & 10 & 10 & 10 & 10 & 9 & 9 \\
\hline 44 & 12.9 & 13 & 11 & 11 & 11 & 10 & 10 & 10 & 10 \\
\hline 45 & 13.4 & 13 & 12 & 11 & 11 & 11 & 11 & 10 & 10 \\
\hline 46 & 13.9 & 14 & 12 & 12 & 12 & 11 & 11 & 11 & 11 \\
\hline 47 & 14.4 & 14 & 13 & 12 & 12 & 12 & 12 & 11 & 11 \\
\hline 48 & 15 & 15 & 13 & 13 & 13 & 12 & 12 & 12 & 12 \\
\hline 49 & 15.6 & 15 & 14 & 14 & 13 & 13 & 13 & 12 & 12 \\
\hline 50 & 16.4 & 16 & 15 & 14 & 14 & 14 & 13 & 13 & 13 \\
\hline 51 & 16.9 & 17 & 15 & 15 & 15 & 14 & 14 & 14 & 13 \\
\hline 52 & 17.7 & 17 & 16 & 16 & 15 & 15 & 15 & 14 & 14 \\
\hline 53 & 18.5 & 18 & 17 & 16 & 16 & 16 & 15 & 15 & 15 \\
\hline 54 & 19.1 & 19 & 17 & 17 & 17 & 16 & 16 & 16 & 16 \\
\hline 55 & 19.9 & 20 & 18 & 18 & 17 & 17 & 17 & 17 & 16 \\
\hline 56 & 20.9 & 21 & 19 & 19 & 18 & 18 & 18 & 18 & 17 \\
\hline 57 & 21.9 & 21 & 20 & 20 & 19 & 19 & 19 & 18 & 18 \\
\hline 58 & 22.8 & 22 & 21 & 20 & 20 & 20 & 20 & 19 & 19 \\
\hline 59 & 23.8 & 23 & 22 & 21 & 21 & 21 & 21 & 20 & 20 \\
\hline 60 & 24.9 & 24 & 23 & 22 & 22 & 22 & 22 & 21 & 21 \\
\hline 61 & 26 & 25 & 24 & 24 & 23 & 23 & 23 & 22 & 22 \\
\hline 62 & 27.2 & 26 & 25 & 25 & 24 & 24 & 24 & 24 & 23 \\
\hline 63 & 28.5 & 28 & 26 & 26 & 26 & 25 & 25 & 25 & 25 \\
\hline 64 & 29.8 & 29 & 27 & 27 & 27 & 27 & 26 & 26 & 26 \\
\hline 65 & 30 & 30 & 29 & 28 & 28 & 28 & 28 & 27 & 27 \\
\hline
\end{tabular}

Table 3: Example of an extrapolated test norm, compared with the real norm. Scaled scores in this subtest have mean $=15$ and $\mathrm{sd}=5$

\subsection{Eliminating floor- and ceiling effects and creating extended norms}

Although some might ask why extended norms are needed, research has shown that differentiating between moderately and highly gifted children reveals important consequences for educational planning (Robertson, Smeets, Lubinski, \& Benbow, 2010). Subtest norm tables typically report scaled scores from $3 \mathrm{SD}$ below to $3 \mathrm{SD}$ above the mean. This results in the fact that, when a child attains the maximum scaled score of +3 SD on one or more of the subtests, both the subtest scores and the composite scores based on these subtests might be underestimating the actual ability level of the child. For the two most recent editions of the popular Wechsler Intelligence Scale for Children (WISC), the WISC-IV and the WISC-V, extended norms have been created for the US edition by extending the original norm tables and using validation samples to confirm that scores for these cases were as expected (Zhu, Cayton, Weis, \& Gabel, 2008; Raiford, Courville, Peters, Gilman, \& Silverman, 2019).

Unfortunately, just like for many other intelligence tests, extended norms are not available for other national standardizations of the WISC-IV and WISC-V, leaving professionals guessing how much the intelligence of their clients is underestimated. Using the DARSIS model, extended norms can be created when enough relatively difficult items are present in a subtest. 
Table 4 is an example of an extended norm. In the original norm table, the mean scaled score is 10 with an SD of 3 . The maximum scaled score is 19, so (for example) a child who has just turned six will receive a scaled score of 19 irregardless of answering 23 or 31 items correct. Using the DARSIS model, the norm has been extended so that answering more items correct yields higher scaled scores. Extending the norms was done in the following way:

Firstly, for each raw score $k$, the value of the difficulty parameter $p$ was determined by selecting the value of $p$ that minimizes the following formula:

$$
\sum_{k}\left(f(x, p)-s_{k, x}\right)^{2}
$$

where $f(x, p)$ is formula 1 from page 8 , and $s_{k, x}$ is the scaled score belonging to a raw score of $k$ and an age of $x$ in the original norm table. Note that in order to find a value of $p$ that is as accurate as possible, the entire norm table needs to be used while minimizing function 5 , and not just the ages one wishes to create extended norms for.

After the value of $p$ is established for the raw score $k$, scaled scores can be estimated for different ages using function 1 .

\begin{tabular}{|c|c|c|c|c|c|c|c|c|c|c|c|c|}
\hline Raw Score & 6.167 & 6.5 & 6.833 & 7.167 & 7.5 & 7.833 & 8.167 & 8.5 & 8.833 & 9.167 & 9.5 & 9.833 \\
\hline 0 & 2 & 2 & 1 & 1 & 1 & 1 & & & & & & \\
\hline 1 & 3 & 3 & 2 & 2 & 2 & 2 & 1 & & & & & \\
\hline 2 & 4 & 4 & 3 & 3 & 3 & 2 & 2 & 1 & & & & \\
\hline 3 & 4 & 4 & 4 & 3 & 3 & 3 & 2 & 2 & 1 & 1 & & \\
\hline 4 & 5 & 5 & 4 & 4 & 4 & 3 & 3 & 2 & 2 & 2 & 1 & 1 \\
\hline $\begin{array}{l}4 \\
5\end{array}$ & $\begin{array}{l}5 \\
5\end{array}$ & 5 & $\begin{array}{l}4 \\
5\end{array}$ & 4 & 4 & 4 & 3 & 3 & 2 & 2 & $\begin{array}{l}1 \\
2\end{array}$ & $\begin{array}{l}1 \\
2\end{array}$ \\
\hline 6 & 6 & 6 & 5 & 5 & 5 & 4 & 4 & 3 & 3 & 3 & 2 & 2 \\
\hline 7 & 6 & 6 & 6 & 5 & 5 & 5 & 4 & 4 & 3 & 3 & 3 & 3 \\
\hline 8 & 7 & 7 & 6 & 6 & 6 & 5 & 5 & 4 & 4 & 4 & 3 & 3 \\
\hline 9 & 8 & 7 & 7 & 6 & 6 & 6 & 5 & 5 & 4 & 4 & 4 & 4 \\
\hline 10 & 8 & 8 & 7 & 7 & 7 & 6 & 6 & 5 & 5 & 5 & 4 & 4 \\
\hline 11 & 9 & 9 & 8 & 8 & 7 & 7 & 6 & 6 & 5 & 5 & 5 & 5 \\
\hline 12 & 9 & 9 & 9 & 9 & 8 & 7 & 7 & 6 & 6 & 6 & 5 & 5 \\
\hline 13 & 10 & 10 & 10 & 9 & 9 & 8 & 7 & 7 & 6 & 6 & 6 & 6 \\
\hline 14 & 11 & 10 & 10 & 10 & 10 & 9 & 8 & 7 & 7 & 7 & 7 & 7 \\
\hline 15 & 12 & 11 & 11 & 11 & 11 & 10 & 9 & 8 & 8 & 8 & 7 & 7 \\
\hline 16 & 13 & $\begin{array}{l}11 \\
12\end{array}$ & $\begin{array}{l}11 \\
12\end{array}$ & $\begin{array}{l}11 \\
12\end{array}$ & 11 & 10 & 10 & 9 & 9 & 8 & 8 & 8 \\
\hline 17 & 13 & 12 & 12 & 12 & 12 & 11 & 10 & 10 & 10 & 9 & 9 & 9 \\
\hline 18 & 14 & 13 & 13 & 13 & 12 & 12 & 11 & 10 & 10 & 10 & 10 & 10 \\
\hline 19 & 15 & 14 & 14 & 14 & 13 & 13 & 12 & 11 & 11 & 11 & 11 & 10 \\
\hline 20 & 16 & 15 & 15 & 15 & 14 & 13 & 13 & 12 & 12 & 12 & 11 & 11 \\
\hline 21 & 17 & 16 & 16 & 16 & 15 & 14 & 14 & 13 & 13 & 13 & 12 & 12 \\
\hline 22 & 18 & $\begin{array}{l}10 \\
17\end{array}$ & $\begin{array}{l}10 \\
17\end{array}$ & $\begin{array}{l}10 \\
17\end{array}$ & $\begin{array}{l}15 \\
16\end{array}$ & $\begin{array}{l}14 \\
15\end{array}$ & $\begin{array}{l}14 \\
15\end{array}$ & 14 & 14 & $\begin{array}{l}13 \\
13\end{array}$ & 13 & 13 \\
\hline 23 & 19 & 18 & 18 & 18 & 17 & 16 & 16 & 15 & 15 & 14 & 14 & 14 \\
\hline 24 & 21.5 & $\begin{array}{l}10 \\
19\end{array}$ & $\begin{array}{l}10 \\
19\end{array}$ & $\begin{array}{l}10 \\
19\end{array}$ & 18 & 17 & 17 & 16 & 16 & 15 & 15 & 15 \\
\hline 25 & 22.8 & 21.9 & 21.1 & 20.3 & 19 & 18 & 18 & 17 & 17 & 16 & 16 & 16 \\
\hline 26 & 24 & 23 & 22.2 & 21.4 & 20.7 & 19 & 19 & 18 & 18 & 17 & 17 & 17 \\
\hline 27 & 25.2 & 24.3 & 23.4 & 22.6 & 21.9 & $\begin{array}{c}11.2 \\
\end{array}$ & 20.5 & $\begin{array}{l}10 \\
19\end{array}$ & $\begin{array}{l}10 \\
19\end{array}$ & 18 & 18 & 18 \\
\hline 28 & 26.5 & 25.6 & 24.7 & 23.8 & 23.1 & 22.4 & 21.7 & 21.1 & 20.6 & 19 & 19 & 19 \\
\hline $\begin{array}{l}20 \\
29\end{array}$ & 27.8 & 26.9 & 25.9 & 25.1 & 24.3 & 23.6 & 22.9 & 22.3 & 21.7 & 21.2 & 20.7 & 20.3 \\
\hline 30 & 29.2 & 28.2 & 27.2 & 26.4 & 25.6 & 24.8 & 24.1 & 23.5 & 22.9 & 22.4 & 21.9 & 21.4 \\
\hline 31 & 30.5 & 29.4 & 28.5 & 27.6 & 26.8 & 26 & 25.3 & 24.7 & 24.1 & 23.5 & 23 & 22.6 \\
\hline
\end{tabular}

Table 4: Example of an extended test norm. Scaled scores in this subtest have mean $=10$ and sd $=3$. Scaled scores above 19 are estimated using the DARSIS model.

\subsection{Norming of new tests}

Ever since intelligence tests were first developed they are normed for different groups in order to describe the performance of an individual relative to a reference group. The most common variable for which an intelligence test is normed is age, so that the performance of an individual can be described relative to a reference group of the same age. Such 'normed' scores are often referred to as scaled scores, and are expressed in various ways such as percentiles or normally distributed scaled 
scores (often with a mean of 100 and an SD of 15 for full scale intelligence test scores, and a mean of 10 and an sd of 3 for subtest scores). Although conventional norming methods where the score distribution was estimated separately for each age were typical in the earlier days, some type of continuous norming is nowadays used by most test standardisations.

In conventional norming, the empirical cumulative distribution function of the raw scores in a particular age group is estimated using a large representative sample for each age group. No continuity assumptions are made and the scaled scores corresponding to each raw score are determined independently for each age band. This type of norming method has the disadvantage of being very dependent on sampling errors, which can result in 'jumps' or 'gaps' in the scaled scores across age. Relatively large sample sizes are necessary for each age band to ensure that high quality norms are estimated.

Because the change in raw test scores across age is gradual, virtually all modern tests are normed using some form of a continuous norming process to improve the accuracy of norms (Lenhard \& Lenhard, 2021; Rigby \& Stasinopoulos, 2005) and to be able to use smaller sample sizes (Oosterhuis, van der Ark, \& Sijtsma, 2016). Continuity requirements (for example strictly increasing percentile curves) are also often assumed, violations are sometimes removed using manual smoothing.

For tests for which it is known beforehand that the norms will adhere to the DARSIS model, using the model in the norming process greatly reduces the number of parameters to be estimated, since for each raw score only the corresponding value of $\mathrm{p}$ must be estimated. Using IRT has one special advantage here. Let $\theta, \theta \in(-\infty, \infty)$ be the latent trait (in this case general intelligence $/ g$ ). Then in order to norm the test, the function:

$$
\begin{array}{r}
g: \mathbb{R} \rightarrow \mathbb{R} \\
p=g(\theta)
\end{array}
$$

must be defined. Let $\mathcal{U}$ be the universe of all people and group $\mathcal{A} \subset \mathcal{U}$ such that all the children in $\mathcal{U}$ that are exactly of age $x$ are in group $\mathcal{A}$. Suppose then that the IRT item parameters of the test are calibrated on group $\mathcal{A}$. In order to resolve the arbitrary scale of the latent trait $\theta$, we fix the mean to 0 and the standard deviation to 1 . The calibration of the item parameters than results in item parameters $M_{\mathcal{A}}$. Since all the children in group $\mathcal{A}$ are of the same age, we then have that:

$$
\begin{aligned}
\theta=s & \\
\Rightarrow & \\
g(\theta) & =g(s)=p(s, x)=p(\theta, x) \\
& =\frac{\theta e^{c_{1}+c_{2} x}-c_{3}}{e^{c_{1}+c_{2} x}+1} \quad\left(e^{c_{1}+c_{2} x}+1 \neq 0\right) \\
& =\left(\frac{e^{c_{1}+c_{2} x}}{e^{c_{1}+c_{2} x}+1}\right) \theta-\frac{c_{3}}{e^{c_{1}+c_{2} x}+1} \quad\left(e^{c_{1}+c_{2} x}+1 \neq 0\right) \\
& =a_{1} \theta+a_{2}
\end{aligned}
$$

This would thus mean that for group $\mathcal{A}$, no further coefficients need to be estimated than the item parameters of the test. In reality however, such a norming design would rarely be carried out. 
A much more common design in the standardization and norming of intelligence tests is to randomly select from the population subgroups of $n$ persons for $m$ different age bands, for example 150 children for each age from six to sixteen, which we will call group $\mathcal{B} \subset \mathcal{U}$. Now suppose that a test is separately calibrated on both group $\mathcal{A}$ and group $\mathcal{B}$. Obviously, $M_{\mathcal{A}}$ and $M_{\mathcal{B}}$ would differ. However, due to the (sub)population invariance property of IRT (De Ayala, 2009; DeMars, 2010; Linden, 2010), the ability scale that person abilities are estimated on using $M_{\mathcal{A}}$ is a linear transformation of the ability scale belonging to $M_{\mathcal{B}}$. For clarity, let $\theta_{\mathcal{A}}$ denote an ability on the ability scale belonging to the calibration on group $\mathcal{A}$, and let $\theta_{\mathcal{B}}$ denote an ability on the ability scale belonging to the calibration on group $\mathcal{B}$. Then, owing to the population invariance property:

$$
\theta_{\mathcal{B}}=d_{1}+d_{2} \theta_{\mathcal{A}}
$$

In order to properly norm the test on group $\mathcal{B}$, the function $p=g_{\mathcal{B}}\left(\theta_{\mathcal{B}}\right)$ must be determined. For any child $c$ with age $x_{c}$ and ability $\theta_{\mathcal{B}}^{c}$, the scaled score $s_{c}$ is then given by:

$$
s_{c}=f\left(x_{c}, p_{c}\right)=f\left(x_{c}, g_{\mathcal{B}}\left(\theta_{\mathcal{B}}^{c}\right)\right)
$$

Using equations 7 and 8, we then get that:

$$
\begin{aligned}
p=g_{\mathcal{B}}\left(\theta_{\mathcal{B}}\right) & =g_{\mathcal{A}}\left(d_{1}+d_{2} \theta_{\mathcal{B}}\right) \\
& =a_{1}\left(d_{1}+d_{2} \theta_{\mathcal{B}}\right)+a_{2} \\
& =a_{1} d_{2} \theta_{\mathcal{B}}+a_{1} d_{1}+a_{2} \\
& =b_{1} \theta_{\mathcal{B}}+b_{2} \quad b_{1}, b_{2} \in \mathbb{R}
\end{aligned}
$$

Which is again linear in $\theta$, only now the constants $b_{1}$ and $b_{2}$ are unknown. In a test norming design where each age group is representative of the population they are drawn from, these constants could for example be estimated using regression by requiring that for each age band, the mean and standard deviation of the scaled scores are as close as possible to 0 and 1 respectively.

In fact, the example above holds for any group $\mathcal{S} \subset \mathcal{U}$. In the case where $\mathcal{S}$ is a convenience sample, not randomly drawn from the population, other information needs to be acquired in order to estimate the 2 coefficients that make up the linear transformation from $\theta$ to $p$.

\subsection{Reference ages}

Due to the structure of $f(x, p)$ (function 1), it is easy for each combination of an age $x$ and a scaled score $s$ to calculate a 'reference age', where the same raw score would lead to a scaled score of 0 (and thus indicating average performance for that age). So for some age $x$ and scaled score $s$ we have that the reference age $r_{x, s}$ is given by:

$$
r_{x, s}=\frac{-c_{1}+\log \left(-\frac{p(x, s)+c_{3}}{p(x, s)}\right)}{c_{2}} \quad p<0
$$

where $p(x, s)$ is defined as in function 4 . Note that this does not work for some combinations of $x$ and $s$, since the average raw score does not increase much after age 20 . The maximum reference 
age could thus be considered to be around 20. In figure 7 the reference age is shown against age for several scaled scores.

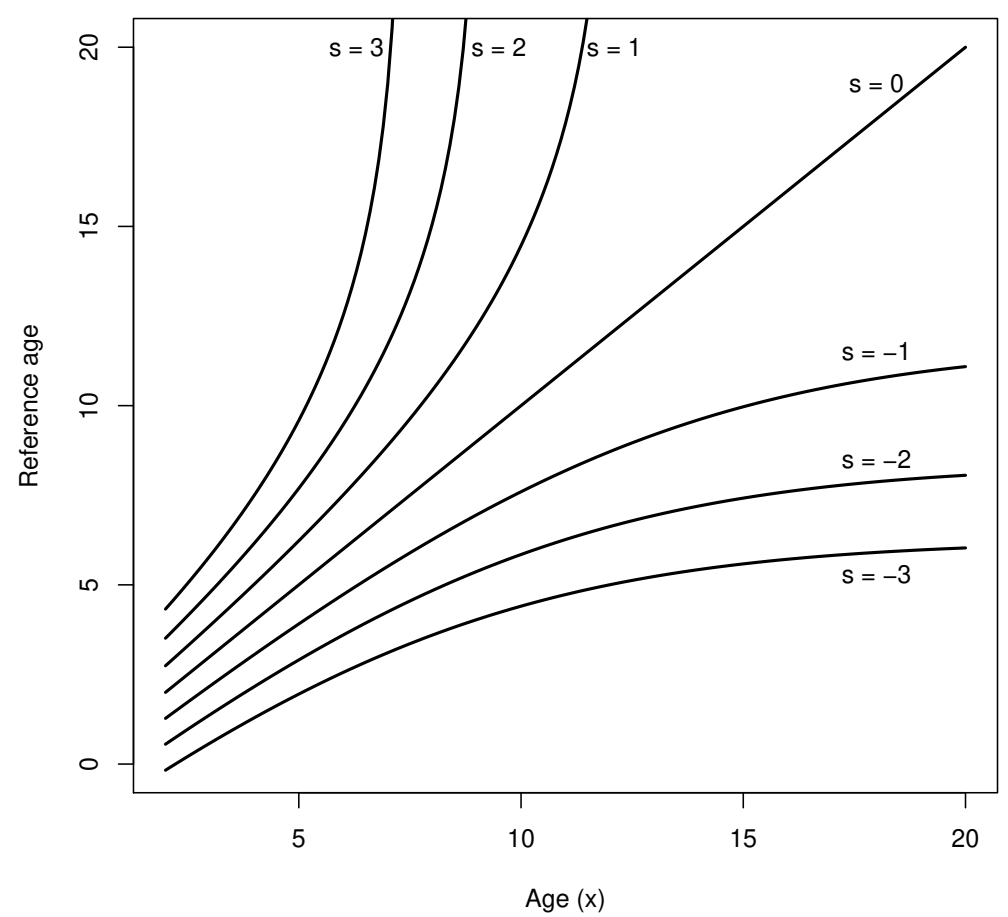

Figure 7: Reference age plotted against age, for several different scaled scores, using function 11

\section{Conclusions}

The structure of how mental reasoning ability evolves from young age to adult is strong and predictable. Almost all of the variance in test norms is explained by the DARSIS model. The model could be used to create test norms for out-of-level testing, to extend test norms, to reduce the parameter space when norming new intelligence tests and to calculate reference ages when not given by a test manual.

\section{References}

De Ayala, R. J. (2009). The theory and practice of item response theory. Guilford Press.

DeMars, C. (2010). Item response theory. Oxford University Press.

Hartshorne, J. K., \& Germine, L. T. (2015). When Does Cognitive Functioning Peak? The Asynchronous Rise and Fall of Different Cognitive Abilities Across the Life Span. Psychological Science, 26(4), 433-443. doi: 10.1177/0956797614567339 
Lenhard, W., \& Lenhard, A. (2021). Improvement of Norm Score Quality via Regression-Based Continuous Norming. Educational and Psychological Measurement, 81(2), 229-261. doi: 10 $.1177 / 0013164420928457$

Li, S.-C., Lindenberger, U., Hommel, B., Aschersleben, G., Prinz, W., \& Baltes, P. B. (2004). Transformations in the Couplings Among Intellectual Abilities and Constituent Cognitive Processes Across the Life Span. Psychological Science, 15(3), 155-163. doi: 10.1111/ j.0956-7976.2004.01503003.x

Linden, W. J. v. d. (Ed.). (2010). Handbook of modern item response theory. Springer.

McArdle, J. J., Ferrer-Caja, E., Hamagami, F., \& Woodcock, R. W. (2002). Comparative longitudinal structural analyses of the growth and decline of multiple intellectual abilities over the life span. Developmental Psychology, 38(1), 115-142. doi: 10.1037/0012-1649.38.1.115

Oosterhuis, H. E. M., van der Ark, L. A., \& Sijtsma, K. (2016). Sample Size Requirements for Traditional and Regression-Based Norms. Assessment, 23(2), 191-202. doi: 10.1177/ 1073191115580638

Raiford, S. E., Courville, T., Peters, D., Gilman, B. J., \& Silverman, L. (2019). WISC$V$ Extended Norms (Tech. Rep. No. 6). The Psychological Corporation. Retrieved from https://www.pearsonassessments.com/content/dam/school/global/clinical/us/ assets/wisc-v/wisc-v-technical-report-6-extended-norms.pdf

Rigby, R. A., \& Stasinopoulos, D. M. (2005). Generalized additive models for location, scale and shape (with discussion). Journal of the Royal Statistical Society: Series C (Applied Statistics), 54(3), 507-554. doi: 10.1111/j.1467-9876.2005.00510.x

Robertson, K. F., Smeets, S., Lubinski, D., \& Benbow, C. P. (2010, December). Beyond the Threshold Hypothesis: Even Among the Gifted and Top Math/Science Graduate Students, Cognitive Abilities, Vocational Interests, and Lifestyle Preferences Matter for Career Choice, Performance, and Persistence. Current Directions in Psychological Science, 19(6), 346-351. doi: $10.1177 / 0963721410391442$

Tucker-Drob, E. M. (2009). Differentiation of cognitive abilities across the life span. Developmental Psychology, 45(4), 1097-1118. doi: 10.1037/a0015864

Warne, R. T. (2012, July). History and Development of Above-Level Testing of the Gifted. Roeper Review, 34(3), 183-193. doi: 10.1080/02783193.2012.686425

Zhu, J., Cayton, T., Weis, L., \& Gabel, A. (2008). WISC-IV Extended Norms (Tech. Rep. No. 7). The Psychological Corporation. Retrieved from https://images.pearsonclinical.com/images/ assets/WISC-IV/WISCIV_TechReport_7.pdf 